\title{
Improved absorption and in vivo kinetic characteristics of nanoemulsions containing evodiamine-phospholipid nanocomplex
}

This article was published in the following Dove Press journal:

International Journal of Nanomedicine

17 September 2014

Number of times this article has been viewed

\section{Jiangbo $\mathrm{Hu}^{1, *}$ \\ Dilong Chen ${ }^{2, *}$ \\ Rong Jiang ${ }^{2}$ \\ Qunyou Tan ${ }^{3}$ \\ Biyue Zhu ${ }^{4}$ \\ Jingqing Zhang'}

'Medicine Engineering Research Center, Chongqing Medical University, Chongqing, People's Republic of China; ${ }^{2}$ Department of Histology and Embryology, Laboratory of Stem Cell and Tissue Engineering, Chongqing Medical University, ${ }^{3}$ Department of Thoracic Surgery, Institute of Surgery Research, Daping Hospital, Third Military Medical University, Chongqing, ${ }^{4}$ West China School of Pharmacy, Sichuan University, Chengdu, People's Republic of China

*These authors contributed equally to this work
Correspondence: Jingqing Zhang Medicine Engineering Research Center, Chongqing Medical University, Room 802, Building 9, Shiyoulu Road I, Yuzhong District, Chongqing 400042, People's Republic of China

Tel +86 I3308300303

Fax +86236848516I

Email zjqrae0I@I63.com
Purpose: The purpose of this study was to assess the improved absorption and in vivo kinetic characteristics of a novel water-in-oil nanoemulsion containing evodiamine-phospholipid nanocomplex (NEEPN) when administered orally.

Methods: NEEPN was fabricated by loading an evodiamine-phospholipid nanocomplex into a water-in-oil nanoemulsive system. The gastrointestinal absorption of NEEPN was investigated using an in situ perfusion method. The modified in vivo kinetic characteristics of evodiamine (EDA) in NEEPN were also evaluated.

Results: Compared with EDA or conventional nanoemulsions containing EDA instead of evodiamine-phospholipid complex, NEEPN with its favorable in vivo kinetic characteristics clearly enhanced the gastrointestinal absorption and oral bioavailability of EDA; for example, the relative bioavailability of NEEPN to free EDA was calculated to be $630.35 \%$, and the effective permeability of NEEPN in the colon was 8.64-fold that of EDA.

Conclusion: NEEPN markedly improved the oral bioavailability of EDA, which was probably due to its increased gastrointestinal absorption. NEEPN also increased efficacy and reduced adverse effects for oral delivery of EDA. Such finding demonstrates great clinical significance as an ideal drug delivery system demands high efficacy and no adverse effects.

Keywords: nanoemulsive system, evodiamine-phospholipid, nanocomplexes, gastrointestinal absorption, oral bioavailability, water-in-oil

\section{Introduction}

Evodiamine (EDA) is a major constituent of the plant Evodia rutaecarpa, and is a traditional Chinese herbal medicine usually taken orally. EDA plays roles in various pharmacological activities, ${ }^{1}$ such as reducing fat uptake, decreasing tissue inflammation, and inhibiting cancer cell proliferation. ${ }^{2,3}$ For EDA, the mechanism of action and extended spectrum of activity have always been areas of interest in medical research. ${ }^{4,5}$ Unfortunately, the use of EDA in clinical applications has been significantly hampered by its reduced bioavailability, mainly due to poor absorption by and availability to targeted tissues. There is an urgent need for suitable EDA delivery systems with enhanced oral bioavailability. However, only a few EDA delivery systems have been reported to date, and most of them were developed for parenteral delivery (such as polymeric magnetic nanocarriers for intravenous delivery, cream for topical use, and microemulsions for transdermal delivery).$^{6-8}$

A water-in-oil nanoemulsion (WNE), sometimes also called a water-in-oil microemulsion, refers to a nanosized system containing two immiscible liquids in which one liquid (water, internal phase) is dispersed in the form of nanosized globules in another liquid (oil, external phase). ${ }^{9} \mathrm{~A}$ WNE usually refers to a clear, isotropic, 
and thermodynamically stable ternary system (water, oil, and a surfactant). Water nanodroplets formed in the bulk oil phase act as a reaction medium for the formation of discrete nanoparticles. A WNE system differs from a conventional emulsive system in its superior features, such as a more attractive appearance (translucent versus opaque), higher dispersion, and stability. ${ }^{9}$ A WNE system differs from an oil-in-water nanoemulsion (or a self-nanoemulsifying drug delivery system, which is essentially an oil-in-water nanoemulsive system) in its built-in properties, such as higher oral absorption, due mainly to higher permeability. In a preliminary study, we found that an EDA-loaded WNE had higher absorption than an EDA-loaded oil-in-water nanoemulsion by comparing the absorption parameters obtained from in situ gastrointestinal perfusion techniques (data not shown). Most WNE systems have been developed for parenteral delivery, such as transdermal delivery of recombinant anthrax protective antigen vaccine for mucosal immunization, intravesical delivery of cisplatin to treat bladder cancer, and transdermal delivery of caffeine for the treatment of skin cancer. ${ }^{10-12}$ In recent studies, WNEs have been produced and used to enhance stability and absorption of peptide drugs, or intestinal permeability of soluble tyrosine kinase inhibitors, which eventually improves their oral bioavailability or cancer treatment efficacy, respectively. ${ }^{13-15}$

Recently, phospholipids have become increasingly important partly due to their potential in improving oral bioavailability and biological efficacy of drugs with low aqueous solubility or low membrane permeability, by forming noncovalently bonded drug-phospholipid complexes. ${ }^{16-19}$ An EDA-phospholipid nanocomplex (EPN) with higher oral bioavailability ( 2.2 -fold that of free EDA) has recently been developed in our laboratory. ${ }^{20}$ EPN showed $~ 3.5$-fold higher hydrophilicity than free EDA. Because some formulations with dissolved the drug-phospholipid complex, such as hydroxysafflor yellow A-phospholipid oil complex and salvianolic acid-phospholipid nanoparticle complex, exhibited superior oral bioavailability over the simple drugphospholipid complex in our preliminary research, ${ }^{21,22}$ we have since tried to further package an insoluble EPN in a WNE nanosystem to achieve added effectiveness.

Although there have been few reports on water-in-oil microemulsive systems developed for the oral delivery of hydrophilic drugs (such as proteins) to date, this is understandable because no water-in-oil nanoemulsive systems have yet been developed for oral delivery of hydrophobic drugs or hydrophobic drug-phospholipid complexes, either. Therefore, the purpose of this study was to assess the improved absorption and in vivo kinetic characteristics of a novel WNE containing evodiamine-phospholipid nanocomplex (NEEPN) when administered orally.

\section{Materials and methods Materials}

EDA was provided by Yuancheng Technology Development Co., Ltd., (Wuhan, People's Republic of China). Soybean phospholipid (Lipoid S 75) was purchased from Phospholipid GmbH (Köln, Germany). Ethyl oleate was provided by Shanghai Chemical Reagent Co., Ltd., (Shanghai, People's Republic of China). Polyethylene glycol 400 (PEG 400) was provided by Chengdu Kelong Chemical Co., Ltd., (Chengdu, People's Republic of China). Cremophor EL 35 (CEL 35) was purchased from BASF Corporation (Ludwigshafen, Germany). All other chemicals and reagents used were of analytical or chromatographic grade.

\section{Preparation and characterization of NEEPN}

NEEPN was obtained by titration stirring methods, and EPN was obtained by modified solvent evaporation methods as described previously. ${ }^{20}$ Briefly, $312.5 \mathrm{mg}$ EDA was added to $250 \mathrm{~mL}$ phospholipid ethanol solution, and maintained at $60^{\circ} \mathrm{C}$ for 3 hours while being continuously stirred by a magnetic stirrer (Type 85-2; Youyi Instruments Co., Ltd., Shanghai, People's Republic of China). The ethanol was subsequently evaporated to dryness under hypobaric conditions. The residue was further dried under vacuum at $40^{\circ} \mathrm{C}$ for 12 hours. After placing the raw product in a desiccation chamber for an additional 12 hours, the dried residue was crushed in a mortar and sieved with a 100 mesh filter $(150 \pm 6.6 \mathrm{~mm})$. The resulting EPN was stored in a desiccation chamber at ambient temperature until use. To prepare NEEPN, EPN was added into a blend of ethyl oleate, CEL 35 and PEG 400 at a mass ratio of 24:13:10, and maintained at $60^{\circ} \mathrm{C}$ for 6 hours while being magnetically stirred. Subsequently, $45 \mathrm{~mL}$ of oil phase (mixture containing EPN, ethyl oleate, CEL 35 and PEG 400) was cooled to $30^{\circ} \mathrm{C}$ under continuous stirring and added dropwise into $5 \mathrm{~mL}$ aliquots of distilled water. The resulting mixture was continuously stirred until the system became translucent, which indicated the formation of NEEPN. A conventional water-in-oil nanoemulsive system containing EDA (CNE) was prepared in a similar way to NEEPN; however, EDA instead of EPN was added to produce CNE.

Diluted NEEPN was prepared by diluting $4 \mathrm{~mL}$ of NEEPN with $16 \mathrm{~mL}$ of ethyl oleate. The conductivity of 
NEEPN and its $4: 1$ dilution were determined at $25^{\circ} \mathrm{C}$ by an electric conductivity analyzer (DDB-303A; Shanghai Precision and Scientific Instrument Co, Ltd, Shanghai, People's Republic of China). The size and zeta potential of neat and diluted NEEPN were determined at $25^{\circ} \mathrm{C}$ by dynamic light scattering (Zetasizer Nano ZS90; Malvern Instruments, Malvern, UK). Our studies were performed at a refractive index of 1.45 , because the refractive index for all formulations being studied is approximately this value.

\section{NEEPN absorption in stomach and intestine}

Animal studies were performed in accordance with the protocol approved by the Laboratory Animal Committee, Chongqing Medical University. Male Sprague Dawley rats weighing $230 \pm 20 \mathrm{~g}$ were all specific pathogen free animals. They were obtained from the Animal Center of Chongqing Medical University (Chongqing, People's Republic of China). During the experimental period, all animals were raised under controlled conditions, and fasted no less than 12 hours before drug administration.

Previously described in situ gastrointestinal perfusion techniques were applied to investigate NEEPN absorption in rats. ${ }^{18,23,24}$ Parenteral anesthesia (3.5\% chloral hydrate) was administered to rats at a dose of $560 \mathrm{mg} / \mathrm{kg}$ via intraperitoneal injection. In a gastric absorption test, the pylorus and cardia of each rat were cannulated with flexible tubing. The stomach was then rinsed with artificial gastric juice. Subsequently, the stomach was perfused with $4 \mathrm{~mL}$ of NEEPN (at the concentration of $400 \mathrm{mg} / \mathrm{mL}$ of EDA), which remained in situ for 2 hours before it was removed. After the perfusion solution was removed, the stomach was rinsed with artificial gastric juice, and the rinsing solution was mixed with the perfusion. The blend of perfusion and rinsing solution was further mixed with a blend of methanol and acetone at a volume ratio of $3: 2$, vortexed for 2 minutes, and centrifuged at 12,000 rpm for 10 minutes. The resulting supernatant was then available for further high performance liquid chromatography (HPLC) analysis. The Elite Hypersil ODS2 C18 HPLC column (Dilian Elite Analytical Instrument Co., Ltd., Dalian, People's Republic of China; $4.6 \mathrm{~mm} \times 250 \mathrm{~mm}, 5 \mu \mathrm{m}$ ) was run at a flow rate of $1 \mathrm{~mL}$ of mobile phase (a mixture of distilled water and methanol at a volume ratio of 25:75) per minute. Effluent was measured at $225 \mathrm{~nm}$. The linearity, precision, and accuracy of the described HPLC method met experimental requirements (data not shown).
In the intestinal absorption test, four intestinal segments of each anesthetized rat were selected, and one end of the chosen enteric sections (each section $10 \mathrm{~cm}$ long) was cannulated with flexible tubing. The start points for cannulation (1 $\mathrm{cm}$ distal to the pyloric sphincter, $15 \mathrm{~cm}$ distal to the pyloric sphincter, $20 \mathrm{~cm}$ proximal to the cecum, and $1 \mathrm{~cm}$ distal to the cecum) were located in the duodenal, jejunal, ileal, and colonic segments, respectively. These intestinal sections were rinsed with physiological saline after being attached to the perfusion assembly, which consisted of a BT100-1L peristaltic pump (Baoding Longer Precision Pump Co, Ltd, Baoding, People's Republic of China). The sections were then equilibrated with Kerbs-Rings solution (SigmaAldrich) ${ }^{18}$ at a flow rate of $0.4 \mathrm{~mL} /$ minute for 15 minutes. Each intestinal segment was perfused with $15 \mathrm{~mL}$ of NEEPN (at the concentration of $400 \mathrm{mg} / \mathrm{mL}$ of EDA) for 1 hour. The flow rate of perfusion solution was $0.2 \mathrm{~mL} / \mathrm{minute}$. After 1 hour, each intestinal segment was rinsed with Krebs Ringer solution (Sigma-Aldrich), and the rinsing solution was mixed with the reserved perfusion. The resulting mixture was processed and analyzed by using the HPLC method described previously in this section.

The absorption parameters of NEEPN in the gastrointestinal tract, such as absorption rate constant $\left(\mathrm{K}_{\mathrm{a}}\right)$, absorption percentages $(\mathrm{PA})$, and effective permeability $\left(\mathrm{P}_{\text {eff }}\right)$, can be calculated in accordance with the following formulas:

$$
\begin{gathered}
\mathrm{K}_{\mathrm{a}}=\left(\mathrm{X}_{0}-\mathrm{X}_{\mathrm{t}}\right) / \mathrm{C}_{0} \mathrm{t} \pi \mathrm{r}^{2} \mathrm{l}, \\
\mathrm{PA}(\%)=\left(\mathrm{X}_{0}-\mathrm{X}_{\mathrm{t}}\right) / \mathrm{X}_{0} \times 100 \%, \text { and } \\
\mathrm{P}_{\text {eff }}=\mathrm{R} \times \ln \left(\mathrm{X}_{\text {in }} / \mathrm{X}_{\text {out }}\right) / 2 \pi \mathrm{rl},
\end{gathered}
$$

where $\mathrm{X}_{0}$ and $\mathrm{X}_{\mathrm{t}}$ are the EDA amount in perfusate at 0 hours and $t$ hours; $\mathrm{C}_{0}$ was the EDA concentration in perfusate at 0 hours, $t$ hours; $X_{\text {in }}$ and $X_{\text {out }}$ are the EDA amounts in inlet and outlet perfusate; $\mathrm{R}$ is the perfusion flow rate; $\mathrm{t}$ is the perfusion time; and $\mathrm{r}$ and 1 are the radius and length of the perfused intestinal segment, respectively. ${ }^{18,23,24}$

\section{In vivo kinetic characteristics of NEEPN}

These animal studies were also performed in accordance with the protocol approved by the Laboratory Animal Committee, Chongqing Medical University (as in the "NEEPN absorption in stomach and intestine" section). Rats were fasted at least 12 hours before drug administration. Each male rat was orally administered NEEPN at a dose of $100 \mathrm{mg} / \mathrm{kg}$. Ophthalmic venous blood samples were collected under ether anesthesia and centrifuged at 3,000 rpm 
for 10 minutes immediately after collection. EDA concentrations in the plasma samples were determined according to the HPLC method of Tan et al. ${ }^{20}$ Honokiol was chosen as the internal standard material. Some experimental data, such as the peak concentration $\left(\mathrm{C}_{\max }\right)$ and peak time $\left(\mathrm{T}_{\max }\right)$, were directly recorded. Primary in vivo kinetic parameters, such as the area under the concentration-time curve (AUC), mean residence time $(\mathrm{MRT})$ and clearance $(\mathrm{Cl})$ were obtained with DAS 2.0 statistical software (Drug and Statistics, Mathematical Pharmacology Professional Committee of China, Shanghai, People's Republic of China). Bioequivalence of NEEPN and EDA (or CNE) was also assessed by running DAS software. Relative bioavailability (RBA) was determined by comparing AUC values after oral administration of equal doses of NEEPN and free EDA (or CNE).

\section{Statistical analysis}

All data are shown as mean \pm standard deviation unless otherwise described. Comparisons between paired groups were performed by using Student's paired $t$-test. Statistical significance was established at probability value $(P$-value $)<0.05$. The $P$-values of the $t$-tests were used to compare measurements obtained from reference and experimental methods, establishing statistical differences at a confidence interval of $95 \%$. In vivo kinetic and bioequivalence analyses were conducted using DAS statistical software.

\section{Results and discussion \\ Design illustration and visual NEEPN}

A detailed description of the appealing features of EPNs has been previously reported by our research group. ${ }^{20}$ Briefly, EPNs show enhanced water solubility ( 3.5 -fold) and oral bioavailability ( 2.2-fold) compared with free EDA. Differential scanning calorimetry, ultraviolet spectroscopy, Fourier transformed infrared spectroscopy, proton nuclear magnetic resonance spectroscopy, and matrix-assisted laser desorption/ionization time-of-flight spectroscopy indicate that EPN is formed by combining EDA and phospholipid through noncovalent bonding. As shown in Figure 1, NEEPN potentially consists of distilled water (water phase), ethyl oleate (oil phase), and interface components (CEL 35, PEG 400 and EPN) ${ }^{20} \mathrm{CEL} 35$, which is amphipathic, has a hydrophilic head and a hydrophobic tail. CEL 35 is a common excipient for injectable and oral use. The manufacturer's material safety data sheet, describes CEL 35 as a low toxicity substance.

In this research, CEL 35 was used as an emulsifier of NEEPN. PEG 400 is soluble not only in water, but also in ethyl oleate. PEG 400 therefore acted as a coemulsifier. Similar to CEL 35, EPN has both hydrophilic and hydrophobic groups. This suggests that in the NEEPN system, EPN might exist in a similar way to CEL 35 . The high volume ratio of oil to water phase (9:1) facilitated formation of stable WNEs. The NEEPN system appeared as a translucent, light yellow color. When NEEPN was diluted with four or nine times the volume of ethyl oleate (oil phase), it remained translucent and in one layer, which further suggests NEEPN is a WNE. There was no significant difference between NEEPN and $\mathrm{CNE}$ in appearance and color. NEEPN was stored at $4^{\circ} \mathrm{C}$ until use. No obvious changes in appearance and conductivity were observed when NEEPN was maintained at $4^{\circ} \mathrm{C}$ for 30 days (data not shown). The parameters (such as the appearance, color and conductivity) were evaluated to determine the stability of the formulation. The translucent NEEPN system was light yellow all the time. No obvious
A

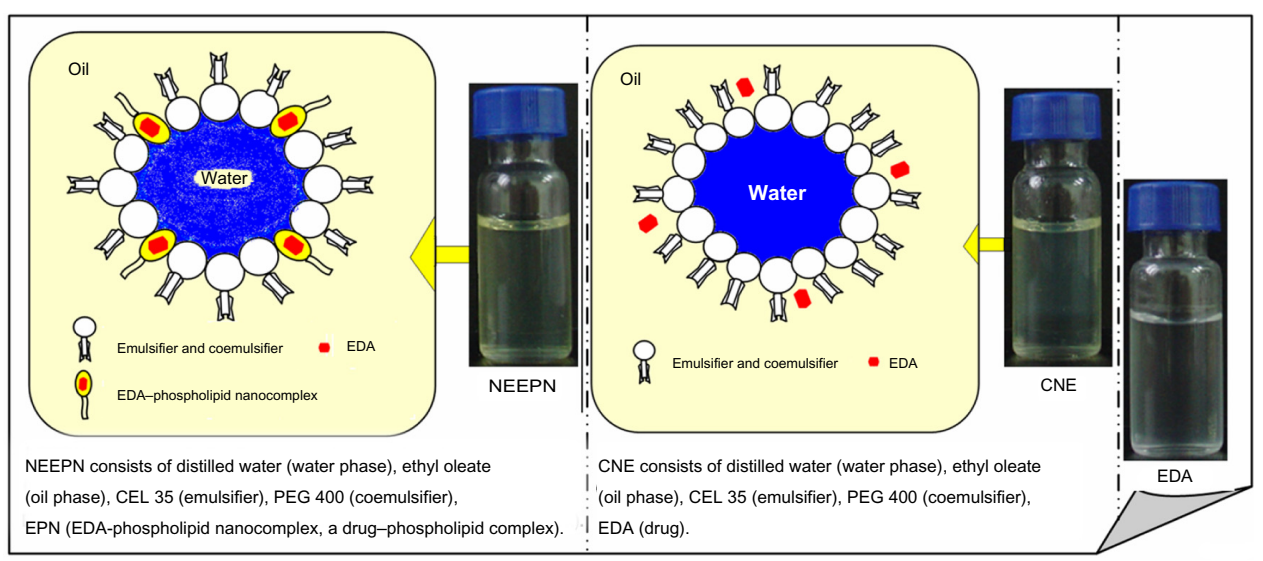

Figure I Schematic diagram and the visual appearance of NEEPN.

Notes: Visual appearance of NEEPN $(\mathbf{A})$ in comparison with CNE (B).

Abbreviations: NEEPN, a water-in-oil nanoemulsive system embedding an evodiamine-phospholipid nanocomplex; CNE, a conventional water-in-oil nanoemulsive system; EDA, evodiamine; CEL 35, cremophor EL 35; PEG 400, polyethylene glycol 400. 
Table I The conductivity of NEEPN and other EDA formulations

\begin{tabular}{lcc}
\hline Formulation & \multicolumn{2}{c}{ Conductivity $(\mu \mathrm{s} / \mathbf{c m})$} \\
\cline { 2 - 3 } & Before dilution & After dilution \\
\hline Free EDA in water & $759.33 \pm 8.50$ & \\
Free EDA in ethyl oleate & $0.37 \pm 0.07$ & \\
CNE without EDA & $12.18 \pm 0.31$ & $0.23 \pm 0.05$ \\
CNE & $12.98 \pm 0.39$ & $0.23 \pm 0.05$ \\
NEEPN without EDA & $16.26 \pm 0.53$ & $0.11 \pm 0.02$ \\
NEEPN & $16.22 \pm 0.27$ & $0.17 \pm 0.05$
\end{tabular}

Notes: A dilution consisted of one part formulation and four parts ethyl oleate. Data presented as mean \pm standard deviation $(n=3)$.

Abbreviations: NEEPN, a water-in-oil nanoemulsive system embedding an evodiamine-phospholipid nanocomplex; EDA, evodiamine; CNE, a conventional water-in-oil nanoemulsive system.

changes were observed when NEEPN was placed at $4^{\circ} \mathrm{C}$ for 30 days.

As seen in Table 1, the conductivity of NEEPN was much higher than that of free EDA in ethyl oleate (oil phase) and slightly higher than that of CNE. However, after dilution as described in the "Preparation and characterization of NEEPN" section, the conductivity of NEEPN decreased to less than half that of CNE. The difference between the conductivities of NEEPN and other EDA formulations indicates the evolving properties of EDA.

NEEPN was diluted with ethyl oleate prior to determining its properties for two reasons: 1) NEEPN is a water-inoil nanoemulsive system embedding an evodiaminephospholipid nanocomplex. The external phase is an oil phase, ie, ethyl oleate; 2) When NEEPN (which appears as a translucent, light yellow color) was mixed with four times the volume of ethyl oleate (oil phase), the mixture remained translucent; when NEEPN was mixed with four times the volume of water (water phase), the mixture soon became very turbid and white.

When the sample cell made of quartz (Malvern Instruments) was used to measure the mean size and zeta potential of the NEEPN dilution (4:1), values were 554.1 $\mathrm{nm}$ and $8.16 \mathrm{mV}$, respectively; when the control sample cell made of general purpose polystyrene (Malvern Instruments) was used, the mean size and zeta potential of NEEPN were $2,789 \mathrm{~nm}$ and $26.3 \mathrm{mV}$, respectively. The reason why the determined size values of the translucent NEEPNs were so high was not clear. In context, there has been no report on the size diameter of a water-in-oil nanoemulsive system used for oral delivery of hydrophobic drug to date, because no water-in-oil nanoemulsive system has been developed for oral delivery of hydrophobic drugs as yet. To the best of our knowledge, few research papers describing WNEs have been published; among them, two research papers describe WNE particle size determination. One report describes the size of a water-in-oil nanoemulsive system used for intravesical delivery of a hydrophobic drug (cisplatin) $;{ }^{11}$ the range of the mean size was $30-90 \mathrm{~nm}$. The mean vesicle size of the nanoemulsions were measured by photon correlation spectroscopy (Nano ZS ${ }^{\circledR}$ 90; Malvern Instruments) using a helium-neon laser with a wavelength of $633 \mathrm{~nm}$. The formulations were diluted five-fold with soybean oil (oil phase of formulation) before measurement. A second report described the droplet diameter of a water-in-oil nanoemulsive system used for transdermal delivery of another hydrophobic drug (caffeine) $;{ }^{12}$ the mean droplet size of caffeine nanoemulsions was found to be in the range of 20.14-105.25 nm. Droplet size distribution of the nanoemulsion was determined by photon correlation spectroscopy using a Zetasizer 1,000 HS (Malvern Instruments).

\section{NEEPN absorption in stomach and intestine}

NEEPN displayed better absorption by the entire gastrointestinal tract, compared to free EDA. The comparison of determined $\mathrm{K}_{\mathrm{a}}, \mathrm{P}_{\text {eff }}$, and PA values between different portions of the gastrointestinal tract (ie, duodenum, jejunum, ileum, and colon), and comparison of those values in different EDA formulations (ie, NEEPN, CNE, and free EDA) are presented in Figures 2 and 3, respectively.

Absorption of free EDA occurred in the stomach at a very low level (PA was less than 5\%). There was as much as a 1.5-fold (or two-fold) increase in gastric absorption for EDA in NEEPN (or CNE) nanosystems.

Recently, intestinal absorption has been recognized as a critical factor affecting the bioavailability of oral drugs. ${ }^{25}$ Unsurprisingly, in our study the intestine was mainly responsible for absorbing EDA. The intestinal absorption of EDA was clearly increased by loading EDA into NEEPN or CNE nanosystems. Furthermore, sites where maximum PA of EDA occurred also changed: among the four intestinal segments, the maximum absorption of free EDA occurred in the duodenum, while for NEEPN, in the jejunum; and for CNE, in the ileum. It should be noted that these differences were not statistically significant. For example, for free EDA, absorption in the duodenum was not statistically different from the jejunum, ileum, or colon. Similar statements could be made about the data for NEEPN and CNE. However, NEEPN had significantly higher $\mathrm{K}_{\mathrm{a}}$ ( or $\mathrm{P}_{\text {eff }}$ ) values than free EDA in every corresponding intestinal section. For ease of comparison, the $\mathrm{K}_{\mathrm{a}}\left(\right.$ or $\left.\mathrm{P}_{\text {eff }}\right)$ value of free EDA in the duodenum was set to 

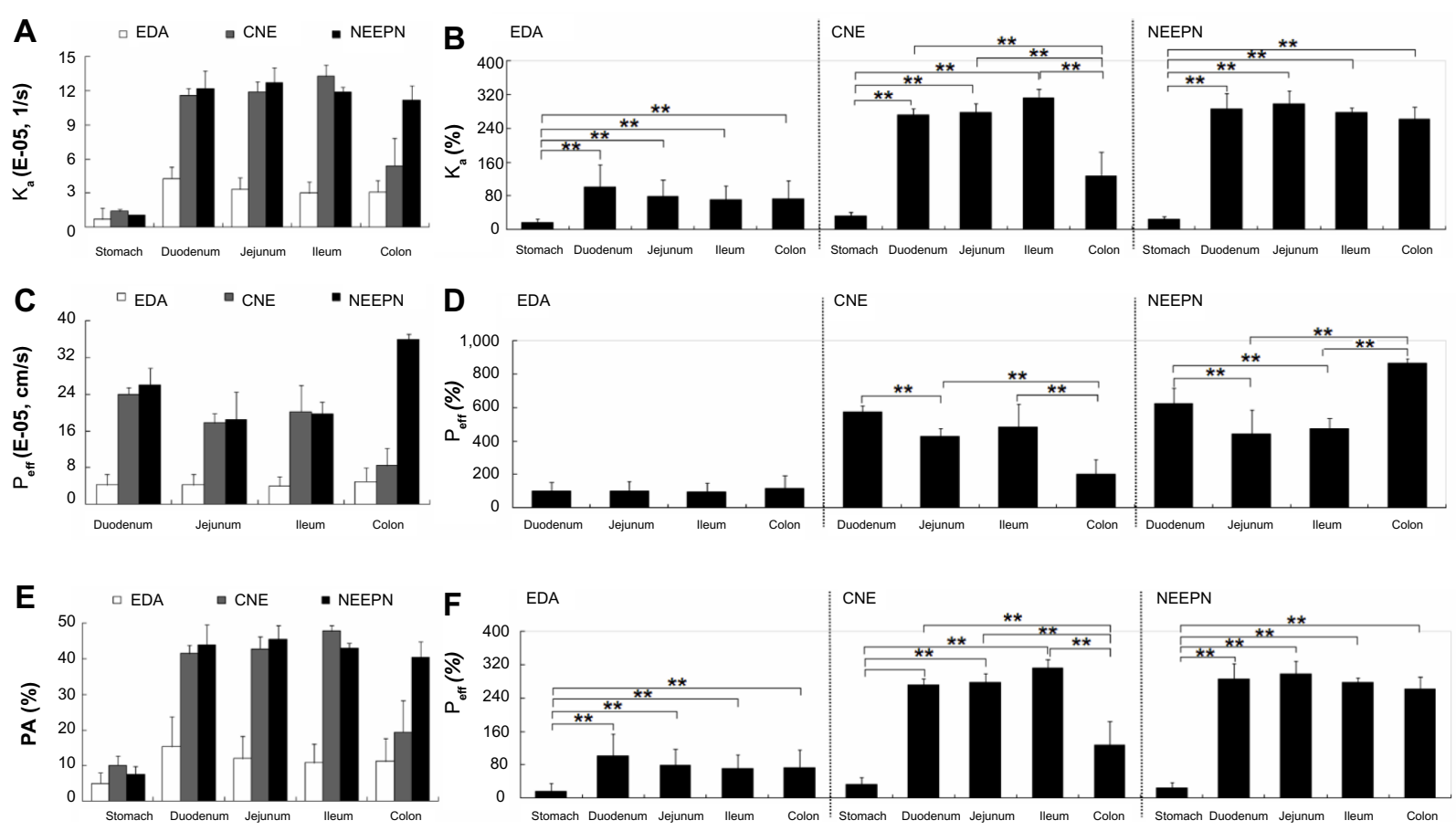

Figure 2 The determined $(\mathbf{A}) \mathrm{K}_{\mathrm{a}},(\mathbf{C}) \mathrm{P}_{\text {eff }}$, and (E) PA values comparison between different portions of the gastrointestinal tract. The calculated (B) $\mathrm{K}_{\mathrm{a}}$ percentages, (D) $\mathrm{P}_{\text {eff }}$ percentages, and (F) PA percentages of NEEPN, CNE and free EDA, compared with that of EDA in the duodenum, respectively.

Notes: Data presented as mean \pm standard deviation $(n=6)$. $* * P<0.01$.

Abbreviations: NEEPN, a water-in-oil nanoemulsive system embedding an evodiamine-phospholipid nanocomplex; CNE, a conventional water-in-oil nanoemulsive system; EDA, evodiamine; $K_{\mathrm{a}}$, absorption rate constant; PA, absorption percentage; $\mathrm{P}_{\text {eff }}$ effective permeability; s, second.
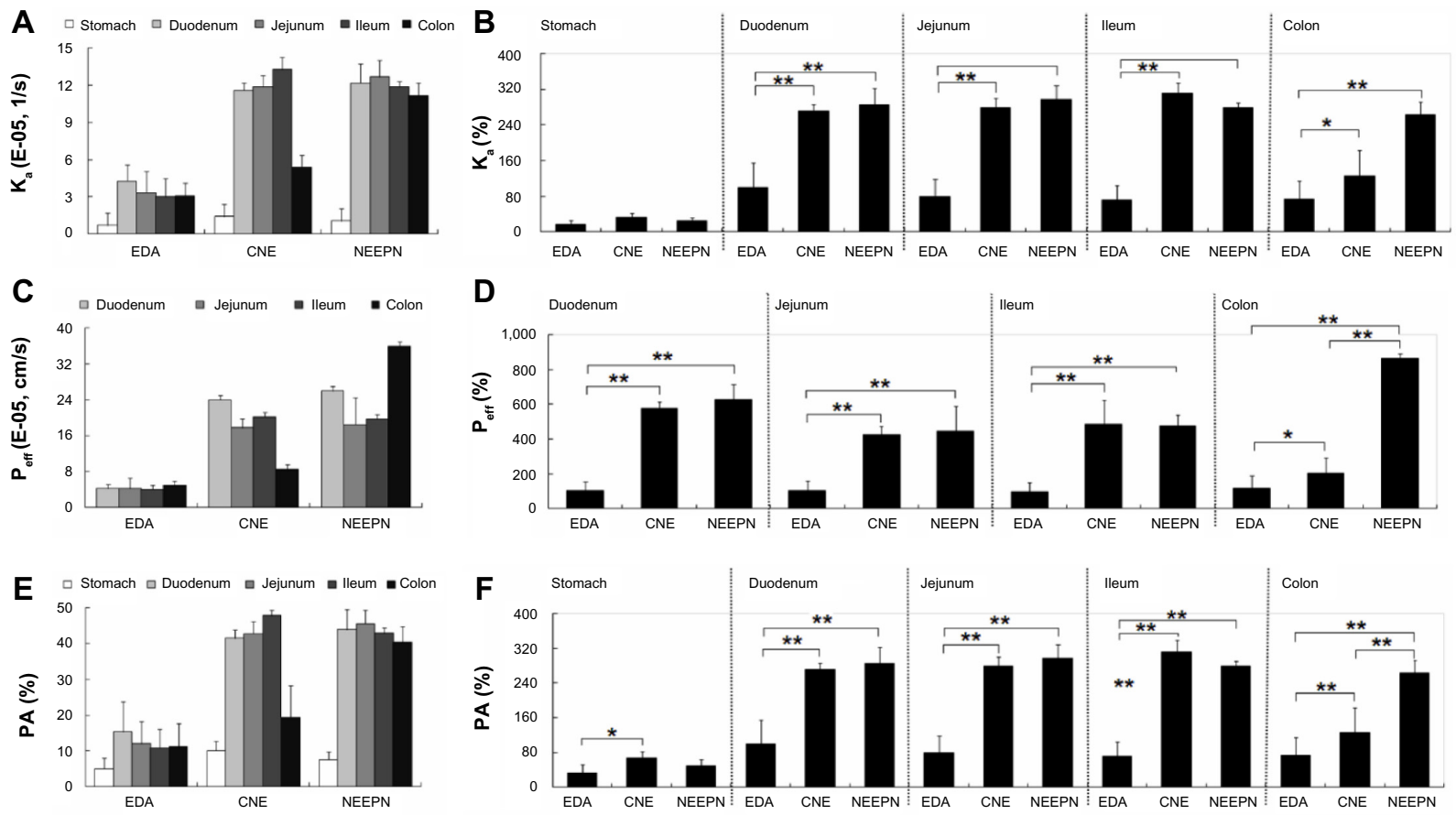

Figure 3 The determined $(\mathbf{A}) \mathrm{K}_{\mathrm{a}},(\mathbf{C}) \mathrm{P}_{\text {eff' }}$ and $(\mathbf{E}) \mathrm{PA}$ values comparison among NEEPN, CNE, and free EDA. The calculated (B) $\mathrm{K}_{\mathrm{a}}$ percentages, (D) $\mathrm{P}_{\text {eff }}$ percentages, and (F) PA percentages of NEEPN, CNE and free EDA, compared with that of EDA in the duodenum, respectively.

Notes: Data presented as mean \pm standard deviation $(n=6)$. $* p<0.05 ; * * p<0.01$.

Abbreviations: NEEPN, a water-in-oil nanoemulsive system embedding an evodiamine-phospholipid nanocomplex; CNE, a conventional water-in-oil nanoemulsive system; EDA, evodiamine; $K_{\mathrm{a}}$, absorption rate constant; PA, absorption percentage; $\mathrm{P}_{\text {eff }}$ effective permeability; s, second. 

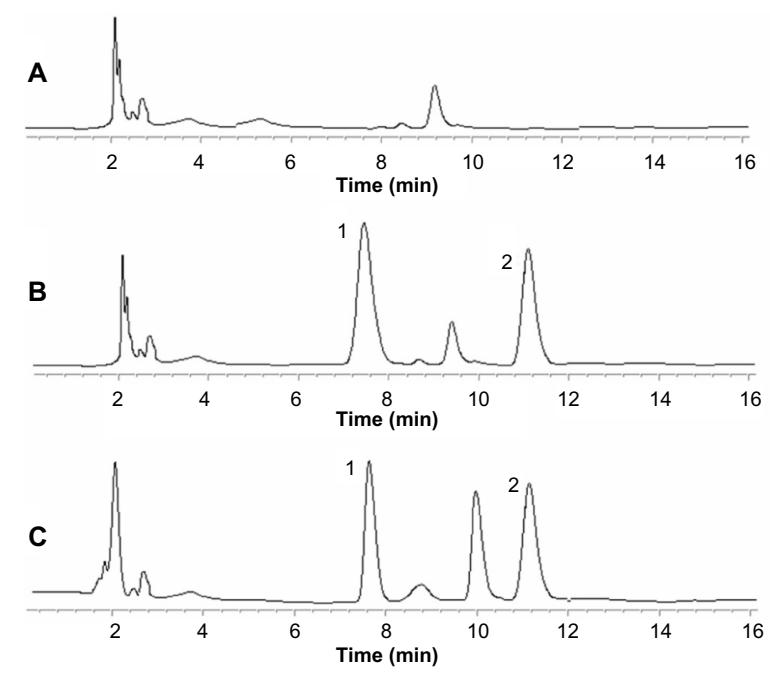

Figure 4 High performance lipid chromatograms of (A) blank plasma; (B) blank plasma spiked with EDA and honokiol, the internal standard; and (C) a sample obtained from rats given NEEPN orally.

Note: Chromatogram peaks labeled (I) are EDA, and (2) honokiol (internal standard).

Abbreviations: EDA, evodiamine; NEEPN, a water-in-oil nanoemulsive system embedding an evodiamine-phospholipid nanocomplex; min, minutes.

$100 \%$; the mean $\mathrm{K}_{\mathrm{a}}\left(\right.$ or $\mathrm{P}_{\text {eff }}$ ) values of NEEPN in the duodenum, jejunum, ileum, and colon were found to increase by $285.71 \%$ (or $624.22 \%$ ), $297.42 \%$ (or $443.17 \%$ ), $278.69 \%$ (or $474.10 \%$ ), and $262.30 \%$ (or $863.79 \%$ ), respectively. In previous research, ${ }^{20}$ mean $\mathrm{K}_{\mathrm{a}}$ (or $\mathrm{P}_{\text {eff }}$ ) values of EPN increased by $231.62 \%$ (or $252.29 \%$ ) in one intestinal segment (including duodenum, jejunum, and ileum). Clearly, there was no statistical difference in the absorption rates of NEEPN between intestinal segments, while there were significant differences between the effective permeabilities of NEEPN in different intestinal segments. The superiority of effective permeabilities of NEEPN in colonic segments was notable. The $\mathrm{K}_{\mathrm{a}}$ ( or $\mathrm{P}_{\text {eff }}$ ) values of CNE were also superior to those of free EDA to a slightly lesser extent in the duodenum and jejunum; to a slightly higher extent in the ileum; and to a much lesser extent in the colon.

The possible causes for superior absorption of NEEPN are: 1) solubilization of EDA by complexing with amphiphilic phospholipid and embedding into the nanoemulsion system; 2) high dispersibility of EDA in the phospholipid nanocomplex and nanoemulsive system; 3 ) protection from enzymatic oxidation (EDA is the substrate of the CYP3A enzyme); ${ }^{26}$ 4) prevention of permeability glycoprotein (Pgp)-mediated EDA efflux (water-insoluble EDA is a Pgp substrate) by embedding EDA into the nanoemulsive system containing constituents such as surfactants and oil phase, which can act as Pgp/CYP450 inhibitors; 5) surfactant-induced or phospholipid-induced membrane fluidity and thus permeability improvement; and 6) the occurrence of intestinal lymphatic transport for drug-phospholipid nanocomplexes and nanoemulsion systems. ${ }^{18,27}$ The reason for the notable superiority of NEEPN absorption in the colon was unclear; however, lymphatic transport might provide an explanation due to the high amounts of lymph fluid in the colon.

\section{In vivo kinetic characteristics of NEEPN}

The typically high performance of lipid chromatograms is presented in Figure 4. A suitable HPLC method to determine

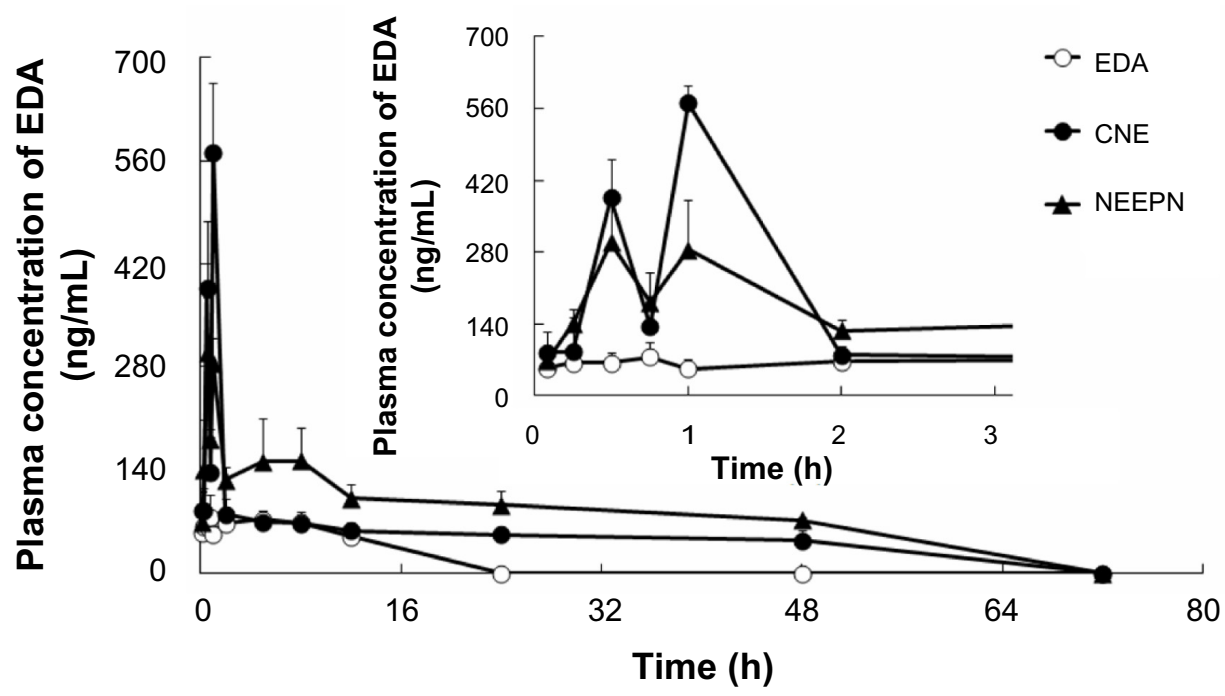

Figure 5 In vivo kinetic profiles (ie, plasma concentration-time curves) of NEEPN and other EDA formulations after oral administration at equal EDA doses of I00 mg/kg, from 0 to 72 hours, and from 0 to 3 hours (inset).

Note: Data presented as mean \pm standard deviation $(n=6)$.

Abbreviations: NEEPN, a water-in-oil nanoemulsive system embedding an evodiamine-phospholipid nanocomplex; CNE, a conventional water-in-oil nanoemulsive system; EDA, evodiamine; $h$, hours. 

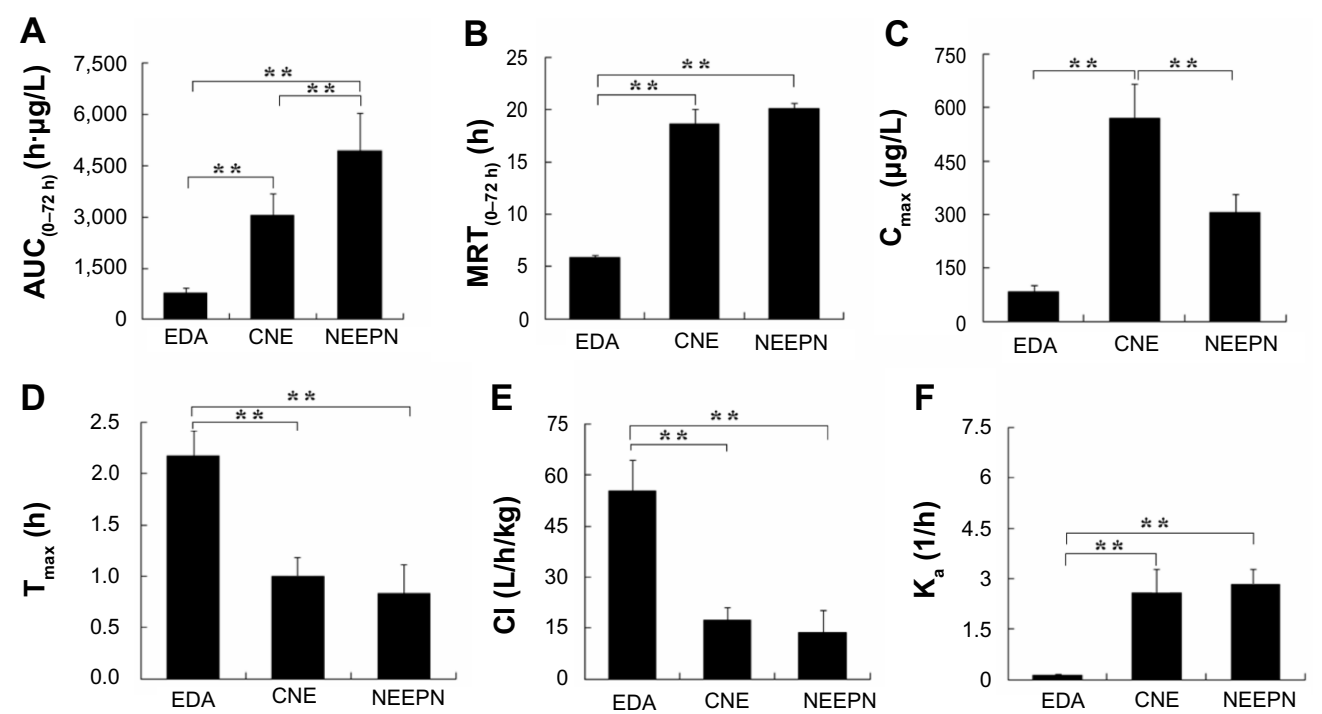

Figure 6 Respective in vivo kinetic parameters of NEEPN and CNE, compared to corresponding parameters of EDA.

Notes: The determined (A) AUC, (B) MRT, (C) $\mathrm{C}_{\text {max }}$, (D) $\mathrm{T}_{\text {max }},(\mathbf{E}) \mathrm{Cl}$, and (F) $\mathrm{K}$ values compared among NEEPN, CNE, and free EDA. $* * P<0.0 \mathrm{I}$.

Abbreviations: NEEPN, a water-in-oil nanoemulsive system embedding an evodiamine-phospholipid nanocomplex; CNE, a conventional water-in-oil nanoemulsive system; EDA, evodiamine; $\mathrm{K}_{2}$, absorption rate constant; $\mathrm{Cl}$, clearance; $\mathrm{T}_{\text {max }}$, peak time; $\mathrm{C}_{\text {max }}$, peak concentration; $\mathrm{MRT}$, mean residence time; $\mathrm{AUC}$, area under the concentration-time curve; h, hours.

the plasma EDA concentrations has been described in detail in the literature. ${ }^{20}$ It was not clear what exactly eluted at 10 minutes in Figure 4C, but because similar peaks appeared at 9.2 minutes and 9.4 minutes in the blank plasma obtained from control rats (Figure 4A) and the blank plasma spiked with EDA and internal standard honokiol (Figure 4B), respectively, the peak at 10 minutes in Figure $4 \mathrm{C}$ might be the background signal produced by the processed blank plasma. The in vivo kinetic curves of NEEPN, CNE, and free EDA (at equal doses of $100 \mathrm{mg} / \mathrm{kg}$ of EDA given orally to rats) are depicted in Figure 5. After administration with NEEPN or CNE, EDA concentrations showed two peaks: one peak was at 0.5 hours and the other peak was at 1 hour. Peak EDA concentrations of CNE were higher than that of NEEPN at the

Table 2 Bioequivalence evaluation of NEEPN and other EDA formulations after oral administration at the same EDA dose of $100 \mathrm{mg} / \mathrm{kg}$

\begin{tabular}{|c|c|c|c|c|c|}
\hline $\begin{array}{l}\text { Formulation I } \\
\text { and } 2\end{array}$ & Parameter & $\begin{array}{l}\text { Calculated } 90 \% \\
\text { confidence interval }\end{array}$ & $\begin{array}{l}\text { Calculated } \\
P \text {-value }\end{array}$ & $\begin{array}{l}\text { Bioequivalence } \\
\text { standard }\end{array}$ & Bioequivalence \\
\hline \multirow{4}{*}{$\begin{array}{l}\text { NEEPN } \\
\text { and EDA }\end{array}$} & AUC & $2,083.5 \%-3,005.5 \%$ & - & $80 \%-125 \%$ & No \\
\hline & $\mathrm{C}_{\max }$ & $32.5 \%-62.5 \%$ & - & $70 \%-143 \%$ & No \\
\hline & $T_{\max }$ & - & $<0.05$ & $>0.05$ & No \\
\hline & $\begin{array}{l}\text { Total bioequivalence } \\
\text { evaluation }\end{array}$ & - & - & - & No \\
\hline \multirow{4}{*}{$\begin{array}{l}\text { NEEPN } \\
\text { and CNE }\end{array}$} & AUC & $107.2 \%-171.1 \%$ & - & $80 \%-125 \%$ & No \\
\hline & $\mathrm{C}_{\max }$ & $156.0 \%-262.6 \%$ & - & $70 \%-143 \%$ & No \\
\hline & $\mathrm{T}_{\max }$ & - & $>0.05$ & $>0.05$ & Yes \\
\hline & $\begin{array}{l}\text { Total bioequivalence } \\
\text { evaluation }\end{array}$ & - & - & - & No \\
\hline \multirow{4}{*}{$\begin{array}{l}\text { CNE } \\
\text { and EDA }\end{array}$} & AUC & $1,319.6 \%-2,338.8 \%$ & - & $80 \%-125 \%$ & No \\
\hline & $\mathrm{C}_{\max }$ & $7.7 \%-37.6 \%$ & - & $70 \%-143 \%$ & No \\
\hline & $\mathrm{T}_{\max }$ & - & $<0.05$ & $>0.05$ & No \\
\hline & $\begin{array}{l}\text { Total bioequivalence } \\
\text { evaluation }\end{array}$ & - & - & - & No \\
\hline
\end{tabular}

Notes: Data presented as mean \pm standard deviation $(n=6)$. - denotes no data or calculations available.

Abbreviations: NEEPN, a water-in-oil nanoemulsive system embedding an evodiamine-phospholipid nanocomplex; EDA, evodiamine; CNE, a conventional water-in-oil nanoemulsive system; $A \cup C$, area under the concentration-time curve; $C_{\text {max }}$, peak concentration; $T_{\text {max }}$, peak time. 
corresponding time points, within 2 hours of administration. Two hours after administration, the EDA concentrations of NEEPN were much higher than those of CNE and free EDA until 48 hours had elapsed since administration (the last time point when EDA was detectable). Compartmental analysis showed that the EDA concentration-time courses of NEEPN and free EDA could be described by a two-compartment model and a one-compartment model, respectively. In other words, when a rat was given NEEPN, the plasma EDA was considered to be distributed into some organs (with a better blood supply) more rapidly and into other organs (with a lower blood flow) more slowly; when a rat was given free EDA, EDA moved rapidly from blood plasma into other body fluids and tissues.

The comparison of the main in vivo kinetic parameters of NEEPN, CNE, and free EDA are presented in Figure 6. Compared with free EDA, the mean AUC, MRT, $\mathrm{C}_{\max }, \mathrm{T}_{\max }$, and $\mathrm{Cl}$ values of NEEPN (or CNE) increased to $630.35 \%$ (or 389.14\%) for AUC, 344.35\% (or 319.18\%) for MRT, $371.53 \%$ (or $691.65 \%$ ) for $\mathrm{C}_{\max }$, or decreased to $38.25 \%$ (or $46.08 \%$ ) for $\mathrm{T}_{\max }$, and $24.99 \%$ (or $31.27 \%$ ) for $\mathrm{Cl}$, respectively. By comparing AUC values, the RBA of NEEPN to free EDA was calculated to be $630.35 \%$, while the RBA of NEEPN to CNE was $161.99 \%$. The RBA of EPN to free EDA has previously been reported as $218.82 \% .{ }^{20} \mathrm{As}$ has been proven, ${ }^{20}$ bioequivalence of two formulations is acceptable only when their $90 \%$ confidence intervals of AUC and $\mathrm{C}_{\max }$ are within the acceptable bioequivalence range of $0.80-1.25$ limits and 0.70-1.43 limits, respectively, and their $\mathrm{T}_{\max }$ values are not significantly different $(P>0.05)$ when using the Wilcoxon rank sum test. As shown in Table 2, paired EDA delivery systems, ie, NEEPN and CNE, NEEPN and free EDA, CNE and free EDA, were not bioequivalent. NEEPN had the highest bioavailability. MRT represented the average time an EDA molecule stayed in a rat, and the $\mathrm{Cl}$ value was a measurement of the renal excretion ability. We suggest that NEEPN, with its longer MRT and lower $\mathrm{Cl}$ values, has prolonged drug action compared to free EDA and CNE. It should be noted that the differences observed between NEEPN and CNE for both MRT and $\mathrm{Cl}$ are not statistically significant. Oral delivery remains the most preferred and common route of medication administration. NEEPN is capable of efficient oral delivery of EDA. Compared to free EDA and CNE, NEEPN with its higher bioavailability clearly indicates better therapy efficacy, shorter treatment duration, as well as less undesirable adverse effects. The markedly superior bioavailability of NEEPN was most likely due to enhanced gastrointestinal absorption (mainly due to the improved colonic absorption) and decreased clearance from systemic circulation in vivo.

\section{Conclusion}

This study assessed the in vivo absorption and kinetic characteristics of an orally administered nanoemulsion containing EPN. Our study indicated that NEEPN has remarkably enhanced in vivo kinetic characteristics and facilitates improved absorption of EDA. Our study also confirmed that NEEPN had the highest bioavailability compared to free EDA and CNEs, which was most likely due to increased colonic absorption. NEEPNs are therefore promising carriers for oral delivery of EDA as they exhibited improved bioavailability, increased efficacy and reduced adverse effects. Moreover, because no WNEs have been developed for oral delivery of hydrophobic drugs (free drugs, or drug-phospholipid nanocomplexes) to date, this study might suggest clinical applications for NEEPN.

\section{Acknowledgments}

This research was partially supported by grants from the Chongqing Natural Science Foundation(CSCT2012JJB10027), and the Chongqing Education Committee Fund (KJ120321).

\section{Disclosure}

The authors report no conflicts of interest in this work.

\section{References}

1. Wang XX, Zan K, Shi SP, et al. Quinolone alkaloids with antibacterial and cytotoxic activities from the fruits of Evodia rutaecarpa. Fitoterapia. 2013;89:1-7.

2. Rasul A, Yu B, Zhong L, Khan M, Yang H, Ma T. Cytotoxic effect of evodiamine in SGC-7901 human gastric adenocarcinoma cells via simultaneous induction of apoptosis and autophagy. Oncol Rep. 2012;27(5):1481-1487.

3. Xu S, Peng J, Li Y, et al. Pharmacokinetic comparisons of rutaecarpine and evodiamine after oral administration of Wu-Chu-Yu extracts with different purities to rats. J Ethnopharmacol. 2012;139(2):395-400.

4. Vyawahare NS, Hadambar AA, Chothe AS, Jalnapurkar RR, Bhandare AM, Kathiravan MK. Effect of novel synthetic evodiamine analogue on sexual behavior in male rats. J Chem Biol. 2012;5(1):35-42.

5. Wu JY, Chang MC, Chen CS, et al. Topoisomerase I inhibitor evodiamine acts as an antibacterial agent against drug-resistant Klebsiella pneumoniae. Planta Med. 2013;79(1):27-29.

6. Chen F, Li S, Li D, Ding JS. Transdermal behaviors comparisons among Evodia rutaecarpa extracts with different purity ofevodiamine and rutaecarpine and the effect of topical formulation in vivo. Fitoterapia. 2012;83(5):954-960.

7. LvY, Ding G, Zhai J, Guo Y, Nie G, Xu L. A superparamagnetic $\mathrm{Fe}_{3} \mathrm{O}_{4}$-loaded polymeric nanocarrier for targeted delivery of evodiamine with enhanced antitumor efficacy. Colloids Surf B Biointerfaces. 2013;110: 411-418.

8. Zhang YT, Huang ZB, Zhang SJ, et al. In vitro cellular uptake of evodiamine and rutaecarpine using a microemulsion. Int J Nanomedicine. 2012;7:2465-2472.

9. Shakeel F, Shafiq S, Haq N, Alanazi FK, Alsarra IA. Nanoemulsions as potential vehicles for transdermal and dermal delivery of hydrophobic compounds: an overview. Expert Opin Drug Deliv. 2012;9(8): 953-974. 
10. Bielinska AU, Janczak KW, Landers JJ, et al. Mucosal immunization with a novel nanoemulsion-based recombinant anthrax protective antigen vaccine protects against Bacillus anthracis spore challenge. Infect Immun. 2007;75(8):4020-4029.

11. Hwang TL, Fang CL, Chen CH, Fang JY. Permeation enhancercontaining water-in-oil nanoemulsions as carriers for intravesical cisplatin delivery. Pharm Res. 2009;26(10):2314-2323.

12. Shakeel F, Ramadan W. Transdermal delivery of anticancer drug caffeine from water-in-oil nanoemulsions. Colloids Surf B Biointerfaces. 2010;75(1):356-362.

13. Gundogdu E, Karasulu HY, Koksal C, Karasulu E. The novel oral imatinib microemulsions: physical properties, cytotoxicity activities and improved Caco-2 cell permeability. J Microencapsul. 2013;30(2): 132-142.

14. Liu D, Kobayashi T, Russo S, et al. In vitro and in vivo evaluation of a water-in-oil microemulsion system for enhanced peptide intestinal delivery. AAPS J. 2013;15(1):288-298.

15. Wen J, Du Y, Li D, Alany R. Development of water-in-oil microemulsions with the potential of prolonged release for oral delivery of L-glutathione. Pharm Dev Technol. 2013;18(6):1424-1429.

16. Fricker G, Kromp T, Wendel A, et al. Phospholipids and lipid-based formulations in oral drug delivery. Pharm Res. 2010;27(8): 1469-1486.

17. Grattagliano I, Diogo CV, Mastrodonato M, et al. A silybin-phospholipids complex counteracts rat fatty liver degeneration and mitochondrial oxidative changes. World J Gastroenterol. 2013;19(20):3007-3017.

18. Tan QY, Hu NN, Liu GD, et al. Role of a novel pyridostigmine bromidephospholipid nanocomplex in improving oral bioavailability. Arch Pharm Res. 2012;35(3):499-508.
19. Zhang JQ, Zhang ZR, Yang H, Tan QY, Qin SR, Qiu XL. Lyophilized paclitaxel magnetoliposomes as a potential drug delivery system for breast carcinoma via parenteral administration: in vitro and in vivo studies. Pharm Res. 2005;22(4):573-583.

20. Tan Q, Liu S, Chen X, et al. Design and evaluation of a novel evodiamine-phospholipid complex for improved oral bioavailability. AAPS Pharm Sci Tech. 2012;13(2):534-547.

21. Peng Q, Zhang ZR, Sun X, Zuo J, Zhao D, Gong T. Mechanisms of phospholipid complex loaded nanoparticles enhancing the oral bioavailability. Mol Pharm. 2010;7(2):565-575.

22. Wang S, Sun M, Ping Q. Enhancing effect of Labrafac Lipophile WL 1349 on oral bioavailability of hydroxysafflor yellow A in rats. Int $J$ Pharm. 2008;358(1-2):198-204.

23. Samiei N, Mangas-Sanjuan V, González-Álvarez I, et al. Ion-pair strategy for enabling amifostine oral absorption: rat in situ and in vivo experiments. Eur J Pharm Sci. 2013;49(4):499-504.

24. Perioli L, Mutascio P, Pagano C. Influence of the nanocomposite MgAlHTlc on gastric absorption of drugs: in vitro and ex vivo studies. Pharm Res. 2013;30(1):156-166.

25. Luo Z, Liu Y, Zhao B, et al. Ex vivo and in situ approaches used to study intestinal absorption. J Pharmacol Toxicol Methods. 2013;68(2):208-216.

26. Sun HZ, Fang ZZ, Cao YF, Sun XY, Hong M. Investigation of the in vitro metabolism of evodiamine: characterization of metabolites and involved cytochrome p450 isoforms. Phytother Res. 2013;27(5):705-712.

27. Zhang JQ, Liu J, Li XL, Jasti BR. Preparation and characterization of solid lipid nanoparticles containing silibinin. Drug Deliv. 2007;14(6): 381-387.
International Journal of Nanomedicine

\section{Publish your work in this journal}

The International Journal of Nanomedicine is an international, peerreviewed journal focusing on the application of nanotechnology in diagnostics, therapeutics, and drug delivery systems throughout the biomedical field. This journal is indexed on PubMed Central, MedLine, CAS, SciSearch ${ }^{\circledR}$, Current Contents ${ }^{\circledR} /$ Clinical Medicine,

\section{Dovepress}

Journal Citation Reports/Science Edition, EMBase, Scopus and the Elsevier Bibliographic databases. The manuscript management system is completely online and includes a very quick and fair peer-review system, which is all easy to use. Visit http://www.dovepress.com/ testimonials.php to read real quotes from published authors. 